\title{
Maraviroc in the treatment of HIV infection
}

\author{
Neelanjana Ray \\ Department of Microbiology, \\ University of Pennsylvania, \\ Philadelphia, PA, USA
}

Correspondence: Neelanjana Ray 301 A Johnson Pavilion, 3610 Hamilton Walk, University of Pennsylvania, Philadelphia PA 19104, USA

Tel + | 215898089 |

Fax +I 2155732883

Email nray@mail.med.upenn.edu

\begin{abstract}
While a successful HIV vaccine will likely take several more years to become a reality, many anti-retroviral (ARV) drugs are currently available to treat HIV infection, and their efficacious use has improved the quality of life and life expectancy of millions of HIV-infected individuals. A recent addition to these ARVs is a new class of drug that targets the HIV entry process by interfering with the action of the CCR 5 coreceptor. The first licensed member of this class is a drug called maraviroc, which is also the first ARV that targets a cellular rather than a viral protein. Several other CCR5 antagonists with varied mechanisms of action are being developed. Key issues with the use of these drugs include determining their potential for use in treatment-naïve versus treatment-experienced patients, the development of sensitive coreceptor phenotyping assays to determine patient eligibility, and finally monitoring the emergence of resistant viruses and their mechanisms of resistance. This review summarizes the preclinical and clinical development of maraviroc as well as studies of HIV resistance to this drug both in vitro and in patients. In addition, a range of diverse CCR5 antagonists currently under development, are also discussed.
\end{abstract}

Keywords: HIV, coreceptor usage, CCR5 inhibitor, maraviroc, drug resistance

\section{Introduction}

Twenty-five years after its discovery, the human immunodeficiency virus (HIV) and its ever-burgeoning prevalence continue to represent a growing worldwide public health problem. According to recent estimates, over 33 million people are living with HIV all over the world (WHO/UNAIDS 2007). In addition, acquired immunodeficiency syndrome (AIDS), the disease caused by HIV, is responsible for over 2 million deaths per annum.

The use of drug cocktails combining multiple compounds that comprise highly active anti-retroviral therapy (HAART) has significantly improved the quality-of-life and life expectancy of millions of HIV-infected individuals. The main components of HAART in the past couple decades have been inhibitors of the viral protease and reverse-transcriptase enzymes. However, in most patients HIV eventually develops resistance to all these drugs. The propensity of the virus for acquiring resistance to any given antiviral agent it is faced with, led to the advent of the combination therapies which constitute HAART, with the paradigm of using three anti-retrovirals in combination, being the current standard of care.

In more recent years, in an effort to target other steps in the virus lifecycle and to develop viable treatments for HAART-resistant HIV patients, entry inhibitors have emerged as a new target for anti-retroviral (ARV) therapy. HIV mediates its entry into target cells using the concerted action of the viral Env protein with a cell surface receptor CD4 and a coreceptor (usually chemokine receptors CCR5 or CXCR4). Viruses that use the CCR5 or the CXCR4 coreceptors alone are called R5 or X4 viruses respectively, while those that can use both are referred to as R5X4 viruses. The Env protein exists as a heterotrimer and is comprised of surface gp120 and transmembrane gp41 subunits. Receptor binding is mediated by gp120 and fusion by gp41. The entry 
process begins with the engagement of the Env trimer on the surface of virions by CD4 on the target cell surface. This is followed by conformational changes that allow coreceptor binding and insertion of the fusion peptide into the target cell membrane. Finally the gp41 protein undergoes dramatic conformational changes, which serve to bring the viral and cellular membranes in close proximity and facilitate membrane fusion.

Entry inhibitors can target the viral entry process, described above, at several steps. These include receptor binding, coreceptor engagement, and membrane fusion. Amongst these candidates the drugs that are farthest along in clinical development include the fusion inhibitors and the CCR5 coreceptor inhibitors.

Enfuvirtide (Fuzeon ${ }^{\circledR} / \mathrm{T}-20$ ) is a peptide fusion inhibitor that targets a conformational intermediate of the fusion process. While enfuvirtide was the only entry inhibitor on the market until late 2007, the use of this drug has been complicated by its need for twice daily injection, leading to some significant problems with regards to compliance and injection site reactions. Owing to these issues, the use of enfuvirtide has been restricted to treatment-experienced patients who are failing HAART, and are on salvage therapy.

CCR5 antagonists are another new class of entry inhibitors under development. These inhibitors block the Env: CCR5 interaction leading to their antiviral effects. Genetic evidence provided strong biological rationale for targeting CCR5 in the development of new entry inhibitors. A mutation in the CCR5 open reading frame results in the premature truncation and a consequent 32-bp deletion in the protein (CCR5 $\Delta 32$ ). Although this mutation is relatively common in the Caucasian population, with an allele frequency of $15 \%-20 \%$, it was found to be significantly underrepresented in the HIV-1 infected groups (Dean et al 1996; Samson et al 1996), and individuals homozygous for the mutation are only rarely infected with HIV (Biti et al 1997; O’Brien et al 1997; Theodorou et al 1997; Michael et al 1998; Gorry et al 2002). In fact, in a group of people at high risk, two individuals that remained uninfected despite repeated exposure were found to be homozygous for the same $\Delta c c r 5$ mutation (Liu et al 1996). Lymphocytes from these individuals are resistant in vitro to R5-using strains but permissive for X4 strains of HIV-1 (Paxton et al 1996). In addition, HIV-1 infected individuals who are heterozygous for the $\Delta c c r 5$ mutation, have around a 2-year delay in their progression to AIDS compared with wildtype controls (Dean et al 1996; Huang et al 1996; Michael et al 1997; Zimmerman et al 1997). Moreover, both heterozygous as well as homozygous carriers of the CCR5 $\Delta 32$ allele were apparently immunocompetent with no obvious abnormalities, suggesting that the absence of CCR5 function might not be harmful and that a CCR5 antagonist should be well tolerated. It should be noted that more recently, an association between lack of CCR5 and an increased susceptibility to West Nile Virus has been reported (Glass et al 2005, 2006), although the mechanistic basis of this observation is not understood.

Most recently in October 2007, maraviroc, the firstin-class CCR5 antagonist, was licensed by the FDA for use in treatment-experienced patients. This review summarizes the recent literature on the use of maraviroc in the treatment of HIV infection as well as the future of CCR5 inhibitors.

\section{Importance of coreceptor usage analysis}

Although HIV can use one of two coreceptors CCR5 or CXCR4 to mediate entry into target cells, upon transmission the majority of newly infected individuals harbor only R5-using viruses. In fact $80 \%$ of ART therapy-naïve patients have only R5-viruses, while $20 \%$ have R5X4 and very few $(<1 \%)$ have X4 viruses (Brumme et al 2005; Moyle et al 2005). Due to conflicting results from different studies, it remains unclear if treated patients (with detectable viremia) maintain similar rates of R5X4 viral prevalence as treatmentnaïve patients (Moyle et al 2005; Hunt et al 2006). However, in highly treatment-experienced patients the prevalence of R5X4 viruses has been shown to approach that of R5 viruses in at least two studies (Melby et al 2006; Wilkin et al 2007). The emergence of X4 using viruses, which usually occurs later in disease, has historically been associated with lowered CD4 cell counts and more rapid progression to disease (Koot et al 1993, 1999; Shankarappa et al 1999). However, it is not clear whether the emergence of $\mathrm{X} 4$ using strains is a cause or an effect of the severe immunodeficiency associated with disease progression to AIDS.

It has been anticipated that the use of CCR 5 antagonists can lead to the emergence of CXCR4-using viruses. While de novo coreceptor switching is observed less commonly, the selection of pre-existing X4 viruses in association with the emergence of resistance to CCR5 antagonists has been reported in several studies. This highlights the importance of accurate and ultrasensitive detection of minority X4-using viruses in a patient's viral quasispecies while determining eligibility for CCR5 antagonist therapy.

Interactions between the HIV Env protein and CD4 lead to the exposure of the coreceptor-binding site, which includes the third variable loop (V3) of gp120 as well as the 
bridging sheet, a discontinuous epitope that is formed only after CD4 engagement. The bridging sheet interacts with the N-terminus of CCR5 or CXCR4. The V3 loop, on the other hand, mediates interactions with the second extracellular loop (ECL2) of CCR5 or CXCR4 and is the principle determinant of coreceptor specificity. In fact specific mutations affecting the charge in the $\mathrm{V} 3$ region have been shown to correlate with coreceptor selectivity. For instance, X4 using viruses usually have higher net positive V3 charge than R5 viruses, which is consistent with the fact that CXCR4 has a lower net positive charge than CCR5. Therefore in principle, env genotypic information should be able to determine the coreceptor usage phenotype.

Several methods have been developed for coreceptor usage prediction based on $\mathrm{V} 3$ region sequence. The simplest of these approaches is the 11/25 rule (De Jong et al 1992; Fouchier et al 1992; Korber et al 1993; Fouchier et al 1995), which predicts that a virus is $\mathrm{X} 4$ using if there are basic amino acids present at positions 11 and 25 of the V3 loop, and R5 using if no basic amino acids present at these positions. While this rule is quite accurate for R5 viruses, it tends to misclassify many X4 using viruses (Jensen et al 2003). Other more sophisticated methods for coreceptor usage prediction, including Webcat, WebPSSM, and geno2-pheno[coreceptor], have recently been reviewed in detail elsewhere (Sierra et al 2007). However, while improvements are being made in this area, the bottomline is that none of these methods have a high enough sensitivity and/or specificity to accurately and consistently predict the tropism of a given Env from its V3 genotype.

Due to the lack of better genotypic predictors, at the moment phenotypic assays based on cell culture experiments, despite their higher cost and slower turnaround time, appear to be the most reliable predictors of coreceptor tropism. Several such approaches have been developed. These include the Trofile assay (Monogram Biosciences), Tropism Recombinant Test (VIRalliance), HIV Phenoscript assay (BioAlliance Pharma) and deCIPhR (inPheno Molecular Diagnostics) among others. However, the most popular one in use at least in the US appears to be the Trofile assay and recently a more sensitive version of this assay called the enhanced Trofile assay has been developed and is being used for detection of X4-using viruses at low levels (Reeves et al 2007).

\section{Maraviroc: preclinical development}

Developed by Pfizer Global Research and Development, maraviroc is the first CCR5 antagonist approved by the FDA for use in treatment-experienced patients harboring only R5 viruses. The drug was identified in a high-throughput screen designed to select compounds that prevented the binding of radiolabeled macrophage inflammatory factor (MIP)-1 $\beta$, an endogenous chemokine, to the CCR5 receptor. The details of the discovery of maraviroc have been reviewed elsewhere (Wood and Armour 2005; Meanwell and Kadow 2007). Briefly, lead optimization efforts focused on improving the binding efficiency of the screening hits and reducing their persistent type 1 CYP2D6 inhibition as well as the potent hERG cardiac potassium channel inhibition liabilities. Eventually, after a lengthy optimization process that entailed the synthesis of almost 1000 compounds, maraviroc was developed. Maraviroc is a potent inhibitor of MIP-1 $\beta$ binding to the CCR5 receptor $\left(\mathrm{IC}_{50}=2 \mathrm{nM}\right)$ and a potent antiviral agent $\left(\mathrm{EC}_{90}=1 \mathrm{nM}\right.$ for inhibition of $\mathrm{HIV}_{\mathrm{BAL}}$ replication in PM1 cells), while its inhibition of the hERG potassium channel is modest (Wood and Armour 2005).

Similar to other small molecular CCR5 inhibitors (discussed below), the mechanism of action of maraviroc is one of allosteric modification. Insertion of these small molecules into a cavity located within the transmembrane helices disrupts the geometry of a multi-point interaction between CCR5 and HIV-1 gp120 (Dragic et al 2000; Tsamis et al 2003; Watson et al 2005; Seibert et al 2006). This multi-point interaction is formed by binding of the second extracellular loop (ECL-2) to elements of the gp120 V3 region and the tyrosine-sulfated N-terminus (Tyr-Nt) of CCR5 binding the more conserved bridging sheet that forms between the $\mathrm{C} 1, \mathrm{C} 2$, and $\mathrm{C} 4$ domains upon CD4 binding (Cormier and Dragic 2002; Huang et al 2007).

\section{In vitro resistance to maraviroc}

Antiretroviral drug resistance is a major hurdle in the success of long-term HIV therapy. HIV has a propensity for acquiring mutations and developing resistance to antiviral drugs owing to its high replication rate coupled with the low fidelity and lack of RT proof-reading resulting in a high error rate. Resistance to entry inhibitors has been a particularly difficult problem since these drugs target the env gene, which is the most variable of all the HIV genes. Specifically, this diversity in env can lead to variable baseline susceptibilities and the pre-existence of resistance mutations in patients naïve to these drugs.

For CCR5 inhibitors such as maraviroc, several possible mechanisms of resistance can be expected (Figure 1). A coreceptor-switching event could occur with R5-using viruses switching to using $\mathrm{CXCR} 4$ or an alternative 


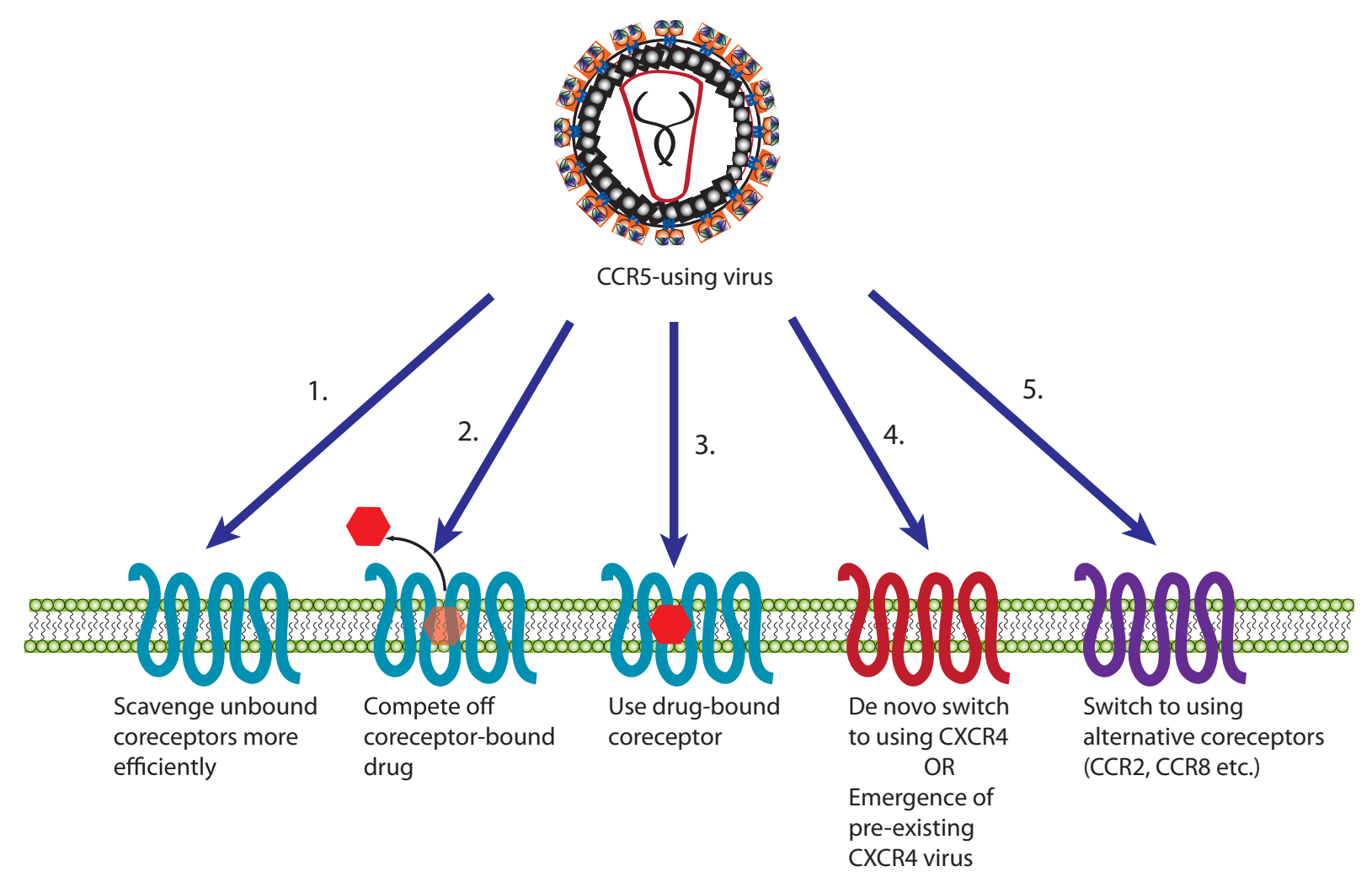

Figure I Potential mechanisms of resistance of HIV to CCR5 antagonists. HIV can become resistant to CCR5 inhibitors in a number of ways. The virus can adapt to scavenge low levels of unbound coreceptors more efficiently either by binding coreceptors with higher affinity or triggering fusion more quickly (I). HIV could also become resistant by competing off drug from coreceptors (2) or by using a drug-bound conformation of the coreceptor (3). Alternatively, the virus could switch to using CXCR4, either via a de novo switch or due to emergence of a pre-existing X4 virus (4), or it could switch to using an alternative coreceptor (5).

coreceptor, or the emergence of pre-existing X4 viruses. Alternatively, viruses could acquire the ability to bind and enter using a drug-bound coreceptor. Resistance to CCR5 inhibitors could also result from viruses that bind coreceptor with higher affinity (and can therefore compete out bound drug), or are able to enter by scavenging low levels of coreceptor either due to higher affinity or a greater proclivity for Env protein triggering.

An in vitro study has shown that maraviroc does not lead to a de novo switch to X4-using viruses during serial passaging of laboratory-adapted and three of the six CCR5-tropic primary isolates studied (Westby et al 2007). However, in the case of one virus (SF162) the emergence of pre-existing X4-using viruses was reported in this study. For two of the passaged primary isolates, maraviroc resistance arose with the mutant Envs acquiring an ability to use a drug-bound form of CCR5. This leads to a characteristic "plateau" of maximal inhibition in a dose-response curve; in other words, beyond a certain maraviroc concentration, increasing drug levels did not inhibit virus infection. These plateaus in dose-response have been observed for viruses resistant to other coreceptor antagonists, suggesting that the recognition of an altered conformation of the coreceptor is a common mechanism for escape from non-competitive inhibitors of entry (Figure 2B). This is in contrast to a competitive mode of resistance that typically leads to clear $\mathrm{IC}_{50}$ shifts in dose-response curves (Figure 2A).

Genotypic changes in resistant viruses are a common hallmark of all classes of anti-retrovirals. Typically mutations accumulate in a step-wise manner in the gene targeted by a drug and either confer resistance or compensate for impaired activity resulting from resistance mutations at the drug target site. For several antiviral agents, the viral genotype is a good measure of phenotype, or the sensitivity of a virus to any given drug. Charts containing mutations that are known to impart resistance to particular drugs are available to physicians, who can create a tailored regimen for each patient based on their viral genotype. In this way molecular information can be used to guide clinical decisions.

In the case of coreceptor antagonists, the V3 loop of gp 120 is the expected site for resistance mutations since this region of the protein is important in mediating the specificity of Envcoreceptor interactions. However, while genotypic changes are found in the V3 region of some coreceptor inhibitor-resistant viruses, in other cases mutations are also observed in other 


\section{A. Competitive Resistance}

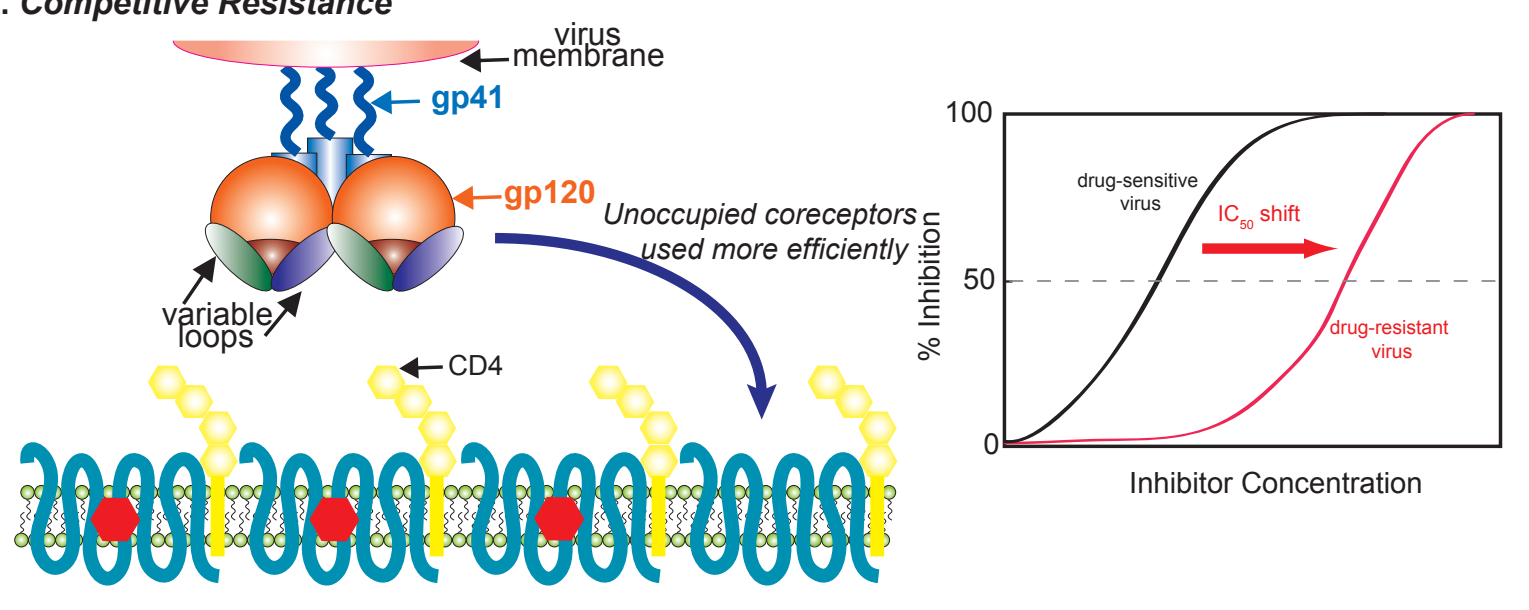

\section{B. Non-competitive Resistance}

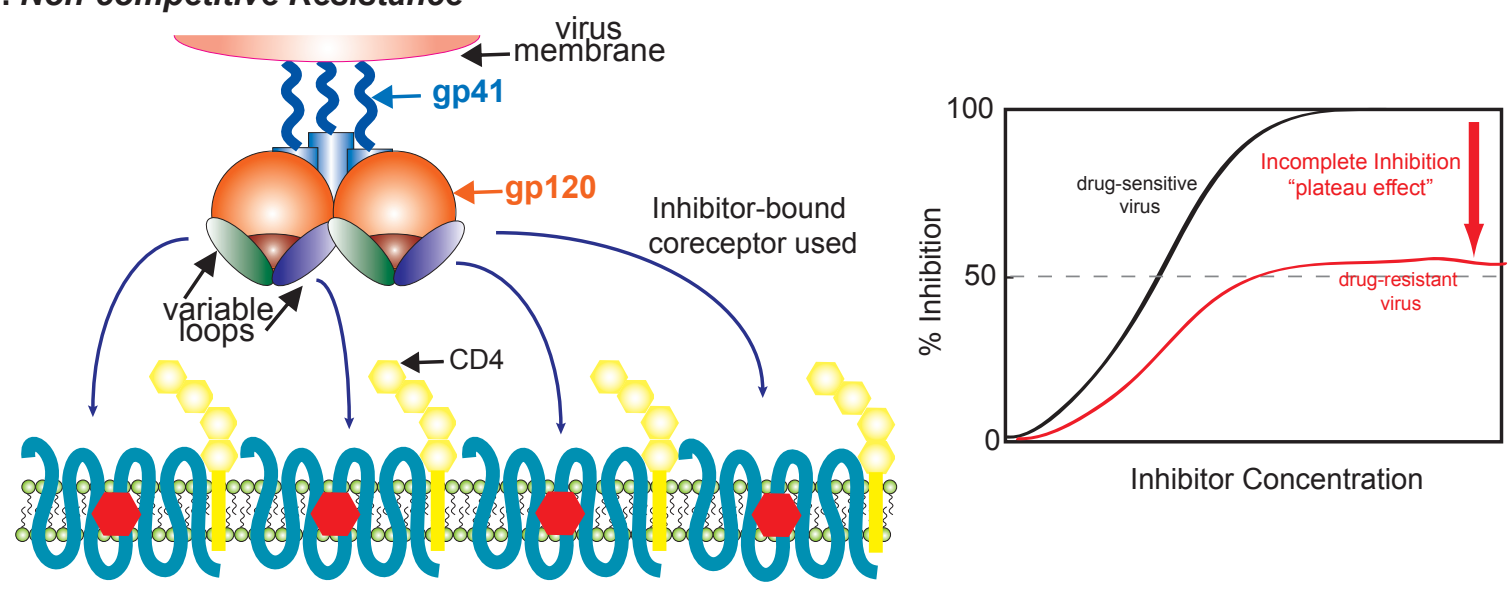

Figure 2 Competitive and non-competitive mechanisms of resistance to coreceptor inhibitors. In a competitive mechanism of resistance (A), HIV acquires the ability to bind unoccupied coreceptors more efficiently, and in this case there is an $\mathrm{IC}_{50}$ shift in a dose response curve relative to a sensitive virus. Alternatively, in a non-competitive mechanism of resistance $(\mathbf{B})$, the virus adapts to enter using a drug-bound conformation of the coreceptor, resulting in a dose-response curve where no further inhibition of virus entry occurs beyond a certain drug concentration ("plateau effect") and no shift in $\mathrm{IC}_{50}$ is observed.

regions of both gp120 and gp41 (Trkola et al 2002; Marozsan et al 2005; Baba et al 2007; Pugach et al 2007; Westby et al 2007; Ogert et al 2008). Moreover, for each parental strain passaged in the presence of coreceptor inhibitors, different resistance-associated changes are observed. Therefore the use of viral env genotype as a predictor of resistance to coreceptor inhibitors such as maraviroc, might be more complicated than for other entry inhibitors such as ENF, where resistance mutations usually map to the HR1 region of gp41 which is the targeted binding site of ENF.

\section{Maraviroc use in the clinic} Phase I and 2 clinical trials

Several phase $1 / 2$ a clinical studies have been conducted with maraviroc, which all showed significant reductions in
HIV viral load in HIV-infected individuals (Fätkenheuer et al 2005). In a study that reviewed data from 5 multipledose, phase $1 / 2$ a double-blinded, placebo-controlled studies of maraviroc, the drug appeared to be well-tolerated in 10-day monotherapy (Fätkenheuer et al 2004). The most adverse effects, which included nausea, rhinitis, dizziness, and headache, were moderate. Some subjects on maraviroc showed elevations in levels of transaminases or occasional elevation in creatinine; however, these effects were not considered to be severe. Postural hypotension was the only dose-limiting complication that occurred at higher rates than placebo in patients receiving a maraviroc dose of $600 \mathrm{mg}$ or higher. Encouragingly, 10-day monotherapy studies with maraviroc resulted in mean viral load reductions of $1.6 \log _{10}$ copies $/ \mathrm{mL}$ with a dose of $300 \mathrm{mg}$ once daily and 
$1.84 \log _{10}$ copies/mL with a dose of $300 \mathrm{mg}$ twice daily. Based on these results, phase $2 b / 3$ studies of maraviroc were initiated in treatment-experienced and treatment-naïve patients.

\section{Maraviroc use in treatment-experienced patients}

MOTIVATE-1 and MOTIVATE-2 were randomized, double-blind placebo-controlled phase $2 \mathrm{~b} / 3$ clinical trials assessing the safety and efficiacy of maraviroc in heavilytreatment experienced patients with triple-class ARV resistance. MOTIVATE-1 included 601 participants from the US and Canada, whereas MOTIVATE-2 included 475 subjects from Europe, Australia and USA. In both studies, subjects were randomly assigned to receive maraviroc at doses of $300 \mathrm{mg}$ once-daily (qd) or twice-daily (bid), or else placebo, in combination with an optimized background therapy (OBT) regimen.

Results from 24 weeks of follow-up in both studies indicated significantly better efficacy in patients treated with maraviroc (qd or bid) + OBT versus placebo + OBT, with the virologic response rates being about twice as high in the maraviroc arms compared to the placebo arm (Lalezari et al 2007; Nelson et al 2007). Specifically, mean decreases in viral load from baseline were $1.95-1.97 \log _{10}$ copies $/ \mathrm{mL}$ in the maraviroc bid arms, $1.82-1.95 \log _{10}$ copies $/ \mathrm{mL}$ in the maraviroc qd arms, and $0.93-1.03 \log _{10}$ copies $/ \mathrm{mL}$ in the placebo arms. $45.6 \%-48.5 \%$ of patients in the maraviroc bid arms, and $40.8 \%-42.2 \%$ in the qd arms achieved viral loads below 50 copies $/ \mathrm{mL}$, compared with $20.9 \%-24.6 \%$ in the placebo arms. Similar trends were observed for viral loads below 400 copies $/ \mathrm{mL}$, indicating that a higher proportion of patients achieved viral loads $<50$ and $<400$ copies $/ \mathrm{mL}$ when maraviroc was administered twice instead of once daily. CD4 cell counts increased from baseline by 102-111 and 107-112 cells $/ \mathrm{mm}^{3}$ in the bid and qd arms, respectively, compared with $52-64$ cells $/ \mathrm{mm}^{3}$ in the placebo arms. While fewer patients in the maraviroc arms experienced treatment failure compared with placebo, more patients in the maraviroc arm experienced a shift in HIV coreceptor usage from CCR5tropic to CXCR4-tropic or dual/mixed tropism. Adverse event profiles were similar in both maraviroc arms and the placebo arm. Based on these 24-week results, in August 2007 the Food and Drug Administration (FDA) approved maraviroc for use in treatment-experienced patients.

More recently, data from a 48-week combined analysis of the MOTIVATE 1 and 2 studies were presented (Hardy et al 2008). As seen in the 24 -week analysis, maraviroc produced greater virological response compared with placebo.
CD4 cell benefits were greater in the maraviroc arms (124 cells $/ \mathrm{mm}^{3}$ bid, 116 cells $/ \mathrm{mm}^{3}$ qd) compared with the placebo arm $\left(61\right.$ cells $\left./ \mathrm{mm}^{3}\right)$. The pooled analysis revealed no new or unique safety findings beyond the 24-week analysis. Discontinuations due to adverse events, serious adverse events, and laboratory abnormalities occurred with similar frequency in the maraviroc and placebo arms. Therefore, treatment with maraviroc appears to provide sustained ARV efficacy and tolerability in treatment-experienced patients.

\section{Maraviroc use in treatment-naïve patients}

Maraviroc in Treatment-naïve Patients (MERIT) is an ongoing phase 3 clinical trial designed to compare the efficacy and safety of maraviroc $300 \mathrm{mg}$ twice daily versus efavirenz $600 \mathrm{mg}$ once daily, each administered in combination with fixed-dose zidovudine/lamivudine $\left(\right.$ Combivir $\left.^{\circledR}\right)$. The study is continuing for 96 weeks; however, preliminary analysis from 48-week data were presented recently (Saag et al 2007; Heera et al 2008). Twice-daily maraviroc was non-inferior to efavirenz in patients with vRNA $<400$ copies $/ \mathrm{mL}(70.6 \%$ vs $73.1 \%)$ but not in the $<50$ copies $/ \mathrm{mL}$ analysis $(65.3 \%$ vs $69.3 \%)$. In subjects with high viral loads (vRNA $>100,000$ copies $/ \mathrm{mL}$ ) the difference was even more pronounced in favor of efavirenz, with the proportion of subjects with $<50$ copies $/ \mathrm{mL}$ on efavirenz $66.6 \%$ and on maraviroc $59.6 \%$. However, maraviroc had a superior safety profile and a more benign lipid profile than efavirenz and an overall higher CD4 benefit.

In another study aimed at analyzing the virological correlates of treatment failure in the MERIT trial, it was found that of 721 patients, 24 (3.3\%) changed from R5 at screening to dual/mixed at baseline (Heera et al 2008). The virologic response in this group of patients was lower both for the efavirenz as well as maraviroc groups, with the proportion of subjects with $<50$ copies $/ \mathrm{mL}$ being only $54.6 \%$ and $7.1 \%$, respectively. Moreover, X4 using viruses were detected at failure in 10/32 (31.3\%) maraviroc patients with R5 virus at baseline. Therefore the conclusions from this study were that the presence of X4 using viruses at baseline is an important predictor of virologic failure on maraviroc. Also, similar to treatment-experienced patients, failure due to the emergence of X4-using virus is an important, albeit infrequently observed, mechanism associated with maraviroc failure.

\section{Future of CCR5 inhibition}

Besides maraviroc, several promising strategies for CCR5 blockade are currently being employed in the development 
of CCR5 antagonists. These include other small-molecule inhibitors, monoclonal antibodies, and genetically derived molecules. Modified chemokine derivates (analogs of the CCR5 ligand RANTES) are also being developed for potential use as microbicides (Kish-Catalone et al 2006; Lusso 2006). In general, CCR5 antagonists can be classified into three broad categories (Figure 3). First, small molecule inhibitors cause allosteric modifications in CCR5, leading to the induction or stabilization of a conformation of CCR5 that can not be bound by gp120, thereby preventing virus entry. Second, monoclonal antibodies sterically block access of virus to CCR5. Finally, genetically derived molecules (zinc finger nucleases) as well as chemokine analogs induce the intracellular trapping of CCR5 to the endoplasmic reticulum, preventing CCR5 expression on the surface of lymphocytes.

Different types of CCR5 inhibitors have their advantages and disadvantages. For instance, small-molecule inhibitors are orally bioavailable, while monoclonal antibodies need to be injected intravenously. On the other hand, monoclonal antibodies usually have prolonged serum half-lives allowing for relatively infrequent administrations, whereas orally administered small molecules need more frequent dosing to maintain adequate concentrations in the face of serum protein binding and active catabolic mechanisms. The therapies discussed below are not intended as an exhaustive summary of all CCR5 inhibitors under development, but rather a sampling of the same to give the reader a flavor for the diversity of potential future options in this drug class.

\section{Allosteric modulation: small molecule inhibitors}

\section{Vicriviroc}

Vicriviroc is the second most advanced agent in the CCR5 antagonist class of ARV drugs. Forty-eight week data from the Phase II VICTOR-E1 study (Vicriviroc in Combination Treatment with Optimized ART Regimen in Experienced Subjects) that examined the safety and efficacy of vicriviroc (30 $\mathrm{mg}$ or $20 \mathrm{mg} \mathrm{qd}$ ) in combination with an optimized ritonavir-boosted, protease inhibitor-containing ART regimen, were recently presented (Zingman et al 2008). Potent and sustained viral suppression was achieved in the vicriviroc arms with 1.77 and $1.75 \log _{10}$ copies/mL mean decreases from baseline viral load compared with $0.79 \log _{10}$ copies $/ \mathrm{mL}$ in the control group. Significantly more patients (56\%) who added vicriviroc $30 \mathrm{mg}$ once daily to a new OBT had fully suppressed HIV-RNA down to $<50$ copies/mL compared with patients on OBT alone (14\%). Also mean increases from baseline in CD4 cell counts in the vicriviroc groups were +102 and +134 cells $/ \mathrm{mm}^{3}$, respectively, compared with +65 in the control group. Moreover, there were no significant differences in the safety profile between the vicriviroc and control arms with respect to liver toxicity, opportunistic infections, malignancies or other conditions. Based on the VICTOR-E1 results, 2 large global phase III trials (VICTOR-E3 and VICTOR-E4) are currently enrolling approximately 375 treatment-experienced subjects (at more than 160 sites worldwide) who will receive $30 \mathrm{mg}$ vicriviroc qd in combination with OBT or OBT alone.

\section{Second-generation maraviroc}

Following the successful licensing of maraviroc, Pfizer is developing a second-generation CCR5 inhibitor. Their lead candidate PF-232798 is an imidazopiperidine CCR5 inhibitor that was identified in a medicinal chemistry synthetic campaign guided by a biological screening cascade after the identification of the triazole inhibitor maraviroc (Dorr et al 2008). PF-232798 is a potent oral CCR5 antagonist with a primary and selectivity/safety pharmacological profile similar to maraviroc. It also has broad-spectrum anti-HIV-1 activity similar to maraviroc. PF-232798 is also active against lab-generated maraviroc-resistant CCR5 tropic HIV-1. The drug binds to the same pocket as maraviroc within the transmembrane region of CCR5, but also shows interactions with the ECL2 hinge region. PF-232798 was well tolerated in normal volunteers and exhibited more favorable pharmacokinetic profile than maraviroc, highlighting its potential for once-daily dosing.

\section{Steric blockade: monoclonal antibodies}

Several antibody-based agents targeting the HIV entry process are being developed. For example, TNX-355 targets CD4, PRO 542 is aimed at gp120, and PRO 140 and HGS004 bind CCR5.

\section{PRO I 40}

PRO 140 is a humanized monoclonal antibody that binds to CCR5 without altering its structure or normal function; it sterically blocks binding of the coreceptor to virus. Interestingly, viral mutants resistant to small-molecule inhibitors (derived by in vitro passage), appear to remain susceptible to inhibition by PRO 140 (Kuhmann et al 2004; Marozsan et al 2005). PRO 140 can also act synergistically with maraviroc and vicriviroc to block membrane fusion mediated by a primary R5 virus (Murga et al 2006). 
PRO 14 was humanized from the original mouse monoclonal antibody PA14 and entered phase $1 \mathrm{~b}$ clinical trials in 2007. In this single-dose study, PRO 140 was well tolerated at all doses and produced significant reductions in viral load and suppressed viral replication for 2-3 weeks (Progenics). PRO 140 has been granted Fast Track designation by the FDA.

\section{HGSO04 (CCR5mAb004)}

HGS004 is a fully human IgG4 monoclonal antibody against CCR5 with robust in vitro activity against several HIV-1 isolates (Lalezari et al 2008). A phase I clinical trial examined the safety and preliminary antiviral activity of this antibody. This was a single-blind, placebo-controlled study that enrolled 63 subjects randomized into 5 dosage cohorts $(0.4,2,8,20$, and $40 \mathrm{mg} / \mathrm{kg})$ who received a single intravenous dose of HGS004 or placebo. HGS004 was well tolerated even at the highest dose and highlevels of receptor occupancy were observed for up to 28 days in the higher dose cohorts. On day $14,54 \%$ of subjects in the 8-, 20- and 40-mg/kg groups had plasma HIV-1 RNA reductions of $>1 \log _{10}$ copies $/ \mathrm{mL}$. In the $40 \mathrm{mg} / \mathrm{kg}$ group, 4 of 10 subjects had a $>1 \log _{10}$ copies/mL plasma RNA reduction at day 28 .

\section{Removal of CCR5 from cell surfaces}

\section{Chemokine analogs}

The natural ligands of CCR5 have been shown to possess anti-HIV activity in vitro (Cocchi et al 1995). Moreover, increased expression of the CCR5 ligand, MIP-1a/CCL3, in humans (as a result of gene duplication) provides protection from HIV acquisition (Gonzalez et al 2005). Chemokine analogs can be engineered to enhance their anti-HIV activity (reviewed in (Hartley and Offord 2005)). Their mechanism of action involves binding of the chemokine receptor (eg. CCR5), followed by agonist-induced receptor internalization, leading to intracellular sequestration (Figure 3). While N-terminally modified chemokine analogs such as AOP-RANTES (Simmons et al 1997) and PSC-RANTES (Hartley et al 2004) have shown prolonged receptor sequestration and potent anti-HIV activity, these protein molecules are not orally bioavailable and are likely to have poor pharmacokinetics after injection due to aggregation on cell surface proteoglycans. However, these factors do not preclude the development of these chemokine analogs as components of topical microbicides in the prevention of HIV transmission (Moore 2005).

\section{Genetic therapies}

Several anti-HIV gene therapy approaches, that target HIV genes or their products, have also been investigated in the past several years (reviewed in [Rossi et al 2007]). These genetic-based approaches, such as intrakines (Yang et al 1997), degrakines (Coffield et al 2003), and zinc-finger nucleases (ZFNs), aim to create phenotypic knockouts of CCR5.

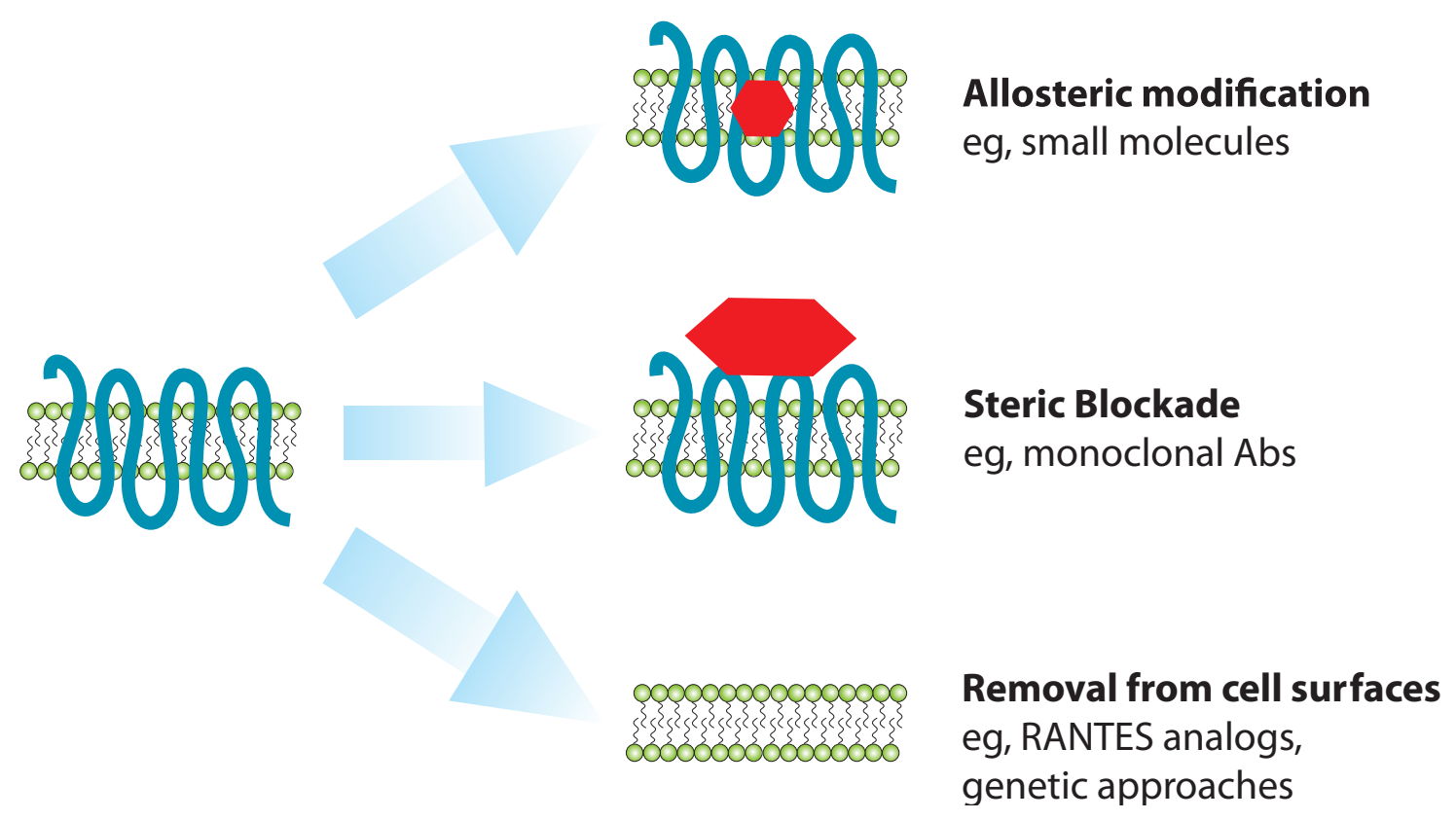

Figure 3 Mechanisms of action of different types of CCR5 inhibitors. Several CCR5 antagonists with diverse modes of action are being developed. In general, there are three categories of these inhibitors: those that allosterically modify CCR5 (such as small molecules), those that sterically block viral access to CCR5 (such as monoclonal antibodies) and finally those that lead to decreased expression of CCR5 on the cell surface (eg, RANTES analogs and genetic therapies such as ZFNs). 
ZFN proteins can be engineered to bind with a high degree of specificity to particular sequence motifs in the genome, and the associated nuclease cleaves the bound DNA (Mani et al 2005). Repair of these double-stranded breaks is associated with the introduction of high frequency deletions and insertions at the cleavage site. CCR5-ZFN proteins have been developed and their administration to T-cell lines and primary human $\mathrm{CD}^{+} \mathrm{T}$-cells resulted in a population of CCR5-modified HIV-resistant cells (Perez et al 2008). ZFNmodified T-cell lines expanded in culture in the presence of HIV, and comprised the majority of cells in the population after 70 days. Genetic disruption of CCR5 imparted robust, stable, and heritable protection against HIV-1 in a NOG/SCID mouse model of infection (Perez et al 2008). The eventual clinical goal is to use these CCR5-ZFNs to perform ex vivo gene therapy on $\mathrm{T}$ cells from HIV-infected individuals and generate a reservoir of permanently HIV-resistant $\mathrm{T}$ cells that can be engrafted back into patients by reinfusion. Therefore, the fact that HIV-infected mice engrafted with ZFN-modified $\mathrm{CD} 4^{+} \mathrm{T}$ cells had lower viral loads and higher $\mathrm{CD} 4^{+} \mathrm{T}$ cell count than mice engrafted with wild-type $\mathrm{CD} 4^{+} \mathrm{T}$ cells, in this study, holds promise for the potential to reconstitute immune function in individuals with HIV/AIDS by maintenance of an HIV-resistant $\mathrm{CD} 4^{+} \mathrm{T}$-cell population.

\section{Conclusion}

Maraviroc is a valuable addition to the arsenal of ARV drugs available in the combat against HIV and AIDS. It heralds a new class of ARV drugs, namely CCR5 inhibitors, with several other second-generation candidates following in the clinical pipeline. While they comprise a promising new approach in controlling HIV infection, CCR5 inhibitors come with their share of problems and important caveats that should be kept in mind for ensuring their successful use. One of the greatest hurdles in the success of this class of drugs is the need to phenotype the patients' viral quasispecies and sensitive detection of very low level CXCR4 usage, to prevent treatment of ineligible patients. In the coming years, more sophisticated coreceptor usage prediction programs as well as phenotypic assays with higher sensitivities are required to overcome this problem.

Resistance is another hurdle that inevitably rears its ugly head in the use of any ARV drug. Coreceptor inhibitors are no exception to this rule. One of the most feared predicted mechanism of resistance to CCR5 inhibitors was a de novo switch to X4-using viruses, which are associated with latestage disease. However, results from most resistance studies, thus far, suggest that this switch to CXCR4 usage is in fact not observed frequently and instead viruses usually acquire resistance to these drugs by using a drug-bound conformation of the CCR5 coreceptor.

Another key issue in the optimal administration of CCR5 antagonists, such as maraviroc, is which patient should receive these drugs: treatment-experienced or treatmentnaïve patients? On the one hand, the use of new ARV drugs finds favor with physicians in a salvage regimen administered to multi-class resistant treatment-experienced patients. In fact this is the current recommendation for the use of maraviroc. However, in the case of CCR5 inhibitors the percentage of patients harboring R5 only using viruses is much higher in treatment-naïve patients (about $80 \%$ in naïve versus $48 \%-60 \%$ in experienced patients), arguing for a more favorable target population in these rather than more experienced subjects. Moreover, maraviroc's new mechanism of action may result in a reduction in the number of latently infected cells, and add to its promise as a candidate for first line therapy. While there is no doubt in the benefit that treatmentexperienced patients (with R5 viruses) can derive from using maraviroc (MOTIVATE trial results), recent clinical data from treatment-naïve patients (MERIT trial) can potentially revitalize interest in CCR5 antagonists as a treatment option for treatment-naïve patients especially since despite its lack of non-inferiority in patients reaching viral loads below 50 copies/mL, maraviroc had a superior safety profile and greater CD4 benefit compared to efavirenz.

Since CCR5 antagonists such as maraviroc target a cellular instead of viral protein (as do all other ARVs), another point to consider in the prescription of these drugs is whether they should be used in addition to triple therapy or as a replacement for one drug of triple therapy. Further studies into the different combinations of maraviroc with existing ARV regimens are required to address this question.

In conclusion, with the advent of maraviroc, an exciting new class of drugs has entered the arena of ARV therapy. Several key issues and questions regarding its optimal use and application remain unanswered, and should be addressed in upcoming clinical trials. Moreover, in the years to come, we can look forward to several other CCR5 antagonists with diverse mechanisms of action becoming available to patients.

\section{Disclosures}

The author reports no conflicts of interest.

\section{References}

Baba M, Miyake H, Wang X, et al. 2007. Isolation and characterization of human immunodeficiency virus type 1 resistant to the small-molecule CCR5 antagonist TAK-652. Antimicrob Agents Chemother, 51:707-15. 
Biti R, Ffrench R, Young J, et al. 1997. HIV-1 infection in an individual homozygous for the CCR5 deletion allele. Nat Med, 3:252-3.

Brumme ZL, Goodrich J, Mayer HB, et al. 2005. Molecular and clinical epidemiology of CXCR4-using HIV-1 in a large population of antiretroviral-naive individuals. J Infect Dis, 192:466-74.

Cocchi F, DeVico AL, Garzino-Demo A, et al. 1995. Identification of RANTES, MIP-1 alpha, and MIP-1 beta as the major HIV-suppressive factors produced by CD8+ T cells. Science, 270:1811-5.

Coffield VM, Jiang Q, Su L. 2003. A genetic approach to inactivating chemokine receptors using a modified viral protein. Nat Biotechnol, 21:1321-7.

Cormier EG, Dragic T. 2002. The crown and stem of the V3 loop play distinct roles in human immunodeficiency virus type 1 envelope glycoprotein interactions with the CCR5 coreceptor. J Virol, 76:8953-7.

De Jong JJ, De Ronde A, Keulen W, et al. 1992. Minimal requirements for the human immunodeficiency virus type $1 \mathrm{~V} 3$ domain to support the syncytium-inducing phenotype: analysis by single amino acid substitution. $J$ Virol, 66:6777-80.

Dean M, Carrington M, Winkler C, et al. 1996. Genetic restriction of HIV-1 infection and progression to AIDS by a deletion allele of the CKR5 structural gene. Hemophilia Growth and Development Study, Multicenter AIDS Cohort Study, Multicenter Hemophilia Cohort Study, San Francisco City Cohort, ALIVE Study. Science, 273:1856-62.

Dorr P, Westby M, McFadyen L, et al. 2008. PF-232798, a second generation oral CCR5 antagonist [abstract]. 15th Conference on Retroviruses and Opportunistic Infections. Boston, MA. Abstract 737.

Dragic T, Trkola A, Thompson DA, et al. 2000. A binding pocket for a small molecule inhibitor of HIV-1 entry within the transmembrane helices of CCR5. Proc Natl Acad Sci USA, 97:5639-44.

Fätkenheuer G, Pozniak A, Johnson M, et al. 2004. Evaluation of dosing frequency and food effect on viral load reduction during short-term monotherapy with UK-427, 857 a novel CCR5 antagonist [abstract]. The XV International AIDS Conference. Bangkok, Thailand. Abstract no. TuPeB4489.

Fätkenheuer G, Pozniak AL, Johnson MA, et al. 2005. Efficacy of shortterm monotherapy with maraviroc, a new CCR5 antagonist, in patients infected with HIV-1. Nat Med, 11: 1170-2.

Fouchier RA, Brouwer M, Broersen SM, et al. 1995. Simple determination of human immunodeficiency virus type 1 syncytium-inducing V3 genotype by PCR. J Clin Microbiol, 33:906-11.

Fouchier RA, Groenink M, Kootstra NA, et al. 1992. Phenotype-associated sequence variation in the third variable domain of the human immunodeficiency virus type 1 gp120 molecule. J Virol, 66:3183-7.

Glass WG, Lim JK, Cholera R, et al. 2005. Chemokine receptor CCR5 promotes leukocyte trafficking to the brain and survival in West Nile virus infection. $J$ Exp Med, 202:1087-98.

Glass WG, McDermott DH, Lim JK, et al. 2006. CCR5 deficiency increases risk of symptomatic West Nile virus infection. $J$ Exp Med, 203:35-40.

Gonzalez E, Kulkarni H, Bolivar H, et al. 2005. The influence of CCL3L1 gene-containing segmental duplications on HIV-1/AIDS susceptibility. Science, 307:1434-40.

Gorry PR, Zhang C, Wu S, et al. 2002. Persistence of dual-tropic HIV-1 in an individual homozygous for the CCR5 Delta 32 allele. Lancet, $359: 1832-4$

Hardy D, Reynes J, Konourina I, et al. 2008. Efficacy and safety of maraviroc plus optimized background therapy in treatmentexperienced patients infected with CCR5-Tropic HIV-1: 48-week combined analysis of the MOTIVATE studies [abstract]. 15th Conference on Retroviruses and Opportunistic Infections. Boston, MA. Abstract 792.

Hartley O, Gaertner H, Wilken J, et al. 2004. Medicinal chemistry applied to a synthetic protein: development of highly potent HIV entry inhibitors. Proc Natl Acad Sci USA, 101:16460-5.

Hartley O, Offord RE. 2005. Engineering chemokines to develop optimized HIV inhibitors. Curr Protein Pept Sci, 6:207-19.
Heera J, Saag MS, Ive P, et al. 2008. Virological correlates associated with treatment failure at week 48 in the phase 3 study of maraviroc in treatment-naive patients [abstract]. 15th Conference on Retroviruses and Opportunistic Infections. Boston, MA. Abstract 40LB.

Huang CC, Lam SN, Acharya P, et al. 2007. Structures of the CCR5 N terminus and of a tyrosine-sulfated antibody with HIV-1 gp120 and CD4. Science, 317:1930-4.

Huang Y, Paxton WA, Wolinsky SM, et al. 1996. The role of a mutant CCR5 allele in HIV-1 transmission and disease progression. Nat Med, $2: 1240-3$.

Hunt PW, Harrigan PR, Huang W, et al. 2006. Prevalence of CXCR4 tropism among antiretroviral-treated HIV-1-infected patients with detectable viremia. $J$ Infect Dis, 194: 926-30.

Jensen MA, Li FS, van't Wout AB, et al. 2003. Improved coreceptor usage prediction and genotypic monitoring of R5-to-X4 transition by motif analysis of human immunodeficiency virus type 1 env V3 loop sequences. J Virol, 77:13376-88.

Kish-Catalone TM, Lu W, Gallo RC, et al. 2006. Preclinical evaluation of synthetic -2 RANTES as a candidate vaginal microbicide to target CCR5. Antimicrob Agents Chemother, 50:1497-509.

Koot M, Keet IP, Vos AH, et al. 1993. Prognostic value of HIV-1 syncytiuminducing phenotype for rate of $\mathrm{CD} 4+$ cell depletion and progression to AIDS. Ann Intern Med, 118:681-8.

Koot M, van Leeuwen R, de Goede RE, et al. 1999. Conversion rate towards a syncytium-inducing (SI) phenotype during different stages of human immunodeficiency virus type 1 infection and prognostic value of SI phenotype for survival after AIDS diagnosis. J Infect Dis, 179:254-8.

Korber BT, Farber RM, Wolpert DH, et al. 1993. Covariation of mutations in the V3 loop of human immunodeficiency virus type 1 envelope protein: an information theoretic analysis. Proc Natl Acad Sci USA, 90:7176-80.

Kuhmann SE, Pugach P, Kunstman KJ, et al. 2004. Genetic and phenotypic analyses of human immunodeficiency virus type 1 escape from a smallmolecule CCR5 inhibitor. J Virol, 78:2790-807.

Lalezari J, Yadavalli GK, Para M, et al. 2008. Safety, pharmacokinetics, and antiviral activity of HGS004, a novel fully human IgG4 monoclonal antibody against CCR5, in HIV-1-infected patients. $J$ Infect Dis, 197:721-7.

Lalezari JP, Goodrich J, DeJesus E, et al. 2007. Efficacy and safety of maraviroc plus optimized background therapy in viremic ART-experienced patients infected with CCR5-tropic HIV-1: 24 week results from a phase 2b/3 study in the US and Canada [abstract]. 14th Conference on Retroviruses and Opportunistic Infections. Los Angeles. Abstract 104bLB.

Liu R, Paxton WA, Choe S, et al. 1996. Homozygous defect in HIV-1 coreceptor accounts for resistance of some multiply-exposed individuals to HIV-1 infection. Cell, 86:367-77.

Lusso P. 2006. HIV and the chemokine system: 10 years later. Embo J, 25:447-56.

Mani M, Kandavelou K, Dy FJ, et al. 2005. Design, engineering, and characterization of zinc finger nucleases. Biochem Biophys Res Commun, 335:447-57.

Marozsan AJ, Kuhmann SE, Morgan T, et al. 2005. Generation and properties of a human immunodeficiency virus type 1 isolate resistant to the small molecule CCR5 inhibitor, SCH-417690 (SCH-D). Virology, 338:182-99.

Meanwell NA, Kadow JF 2007. Maraviroc, a chemokine CCR5 receptor antagonist for the treatment of HIV infection and AIDS. Curr Opin Investig Drugs, 8:669-81.

Melby T, Despirito M, Demasi R, et al. 2006. HIV-1 coreceptor use in triple-class treatment-experienced patients: baseline prevalence, correlates, and relationship to enfuvirtide response. J Infect Dis, 194:238-46.

Michael NL, Chang G, Louie LG, et al. 1997. The role of viral phenotype and CCR-5 gene defects in HIV-1 transmission and disease progression. Nat Med, 3:338-40. 
Michael NL, Nelson JA, KewalRamani VN, et al. 1998. Exclusive and persistent use of the entry coreceptor CXCR4 by human immunodeficiency virus type 1 from a subject homozygous for CCR5 delta 32. J Virol, 72:6040-7.

Moore JP. 2005. Topical microbicides become topical. N Engl J Med, 352:298-300.

Moyle GJ, Wildfire A, Mandalia S, et al. 2005. Epidemiology and predictive factors for chemokine receptor use in HIV-1 infection. $J$ Infect Dis, 191:866-72.

Murga JD, Franti M, Pevear DC, et al. 2006. Potent antiviral synergy between monoclonal antibody and small-molecule CCR5 inhibitors of human immunodeficiency virus type 1. Antimicrob Agents Chemother, 50:3289-96.

Nelson M, Fatkenheuer G, Konourina I, et al. 2007. Efficacy and safety of maraviroc plus optimized background therapy in viremic ARTexperienced patients infected with CCR5-tropic HIV-1 in Europe, Australia and North America: 24 week results [abstract]. 14th Conference on Retroviruses and Opportunistic Infections. Los Angeles. Abstract 104aLB.

O'Brien TR, Winkler C, Dean M, et al. 1997. HIV-1 infection in a man homozygous for CCR5 delta 32. Lancet, 349:1219.

Ogert RA, Wojcik L, Buontempo C, et al. 2008. Mapping resistance to the CCR5 co-receptor antagonist vicriviroc using heterologous chimeric HIV-1 envelope genes reveals key determinants in the C2-V5 domain of gp120. Virology, 373:387-99.

Paxton WA, Martin SR, Tse D, et al. 1996. Relative resistance to HIV-1 infection of CD4 lymphocytes from persons who remain uninfected despite multiple high-risk sexual exposure. Nat Med, 2: 412-7.

Perez E, Wang J, Miller J, et al. 2008. Establishment of HIV-1 resistance in CD4+ T cells by genome editing using zinc-finger nucleases. Nat Biotechnol, 26:808-16.

Progenics PRO 140. Accessed 17 July 2008. URL: http://www.progenics. $\mathrm{com} / \mathrm{pro140.cfm.}$

Pugach P, Marozsan AJ, Ketas TJ, et al. 2007. HIV-1 clones resistant to a small molecule CCR5 inhibitor use the inhibitor-bound form of CCR5 for entry. Virology, 361:212-28.

Reeves JD, Han D, Hunt P, et al. 2007. Enhancements to the Trofile HIV coreceptor tropism assay enable improved detection of CXCR4-using subpopulations and earlier detection of CXCR4-using viruses in sequential patient samples [abstract]. 3rd International Workshop on Targeting HIV Entry. Washington, DC. Abstract 11.

Rossi JJ, June CH, Kohn DB. 2007. Genetic therapies against HIV. Nat Biotechnol, 25:1444-54.

Saag MS, Ive P, Heera J, et al. 2007. A multicenter, randomized, doubleblind, comparative trial of a novel CCR5 antagonist, maraviroc versus efavirenz, both in combination with Combivir (zidovudine [ZDV]/ lamivudine [3TC]), for the treatment of antiretroviral naive patients infected with R5 HIV 1: Week 48 results of the MERIT study. 4th International AIDS Society Conference. Sydney, Australia.

Samson M, Libert F, Doranz BJ, et al. 1996. Resistance to HIV-1 infection in caucasian individuals bearing mutant alleles of the CCR- 5 chemokine receptor gene. Nature, 382:722-5.
Seibert C, Ying W, Gavrilov S, et al. 2006. Interaction of small molecule inhibitors of HIV-1 entry with CCR5. Virology, 349:41-54.

Shankarappa R, Margolick JB, Gange SJ, et al. 1999. Consistent viral evolutionary changes associated with the progression of human immunodeficiency virus type 1 infection. $J$ Virol, 73:10489-502.

Sierra S, Kaiser R, Thielen A, et al. 2007. Genotypic coreceptor analysis. Eur J Med Res, 12:453-62.

Simmons G, Clapham PR, Picard L, et al. 1997. Potent inhibition of HIV-1 infectivity in macrophages and lymphocytes by a novel CCR5 antagonist. Science, 276:276-9.

Theodorou I, Meyer L, Magierowska M, et al. 1997. HIV-1 infection in an individual homozygous for CCR5 delta 32. Seroco Study Group. Lancet, 349:1219-20.

Trkola A, Kuhmann SE, Strizki JM, et al. 2002. HIV-1 escape from a small molecule, CCR5-specific entry inhibitor does not involve CXCR4 use. Proc Natl Acad Sci USA, 99:395-400.

Tsamis F, Gavrilov S, Kajumo F, et al. 2003. Analysis of the mechanism by which the small-molecule CCR5 antagonists SCH-351125 and SCH350581 inhibit human immunodeficiency virus type 1 entry. $J$ Virol, 77:5201-8.

Watson C, Jenkinson S, Kazmierski W, et al. 2005. The CCR5 receptor-based mechanism of action of 873140 , a potent allosteric noncompetitive HIV entry inhibitor. Mol Pharmacol, 67:1268-82.

Westby M, Smith-Burchnell C, Mori J, et al. 2007. Reduced maximal inhibition in phenotypic susceptibility assays indicates that viral strains resistant to the CCR5 antagonist maraviroc utilize inhibitor-bound receptor for entry. $J$ Virol, 81:2359-71.

WHO/UNAIDS. 2007. AIDS epidemic update. Accessed date: 17 July 2008 URL: http://www.unaids.org:80/en/KnowledgeCentre/HIVData/EpiUpdate/EpiUpdArchive/2007/.

Wilkin TJ, Su Z, Kuritzkes DR, et al. 2007. HIV type 1 chemokine coreceptor use among antiretroviral-experienced patients screened for a clinical trial of a CCR5 inhibitor: AIDS Clinical Trial Group A5211. Clin Infect Dis, 44:591-5.

Wood A and Armour D. 2005. The discovery of the CCR5 receptor antagonist, UK-427,857, a new agent for the treatment of HIV infection and AIDS. Prog Med Chem, 43:239-71.

Yang AG, Bai X, Huang XF, et al. 1997. Phenotypic knockout of HIV type 1 chemokine coreceptor CCR-5 by intrakines as potential therapeutic approach for HIV-1 infection. Proc Natl Acad Sci USA, 94:11567-72.

Zimmerman PA, Buckler-White A, Alkhatib G, et al. 1997. Inherited resistance to HIV-1 conferred by an inactivating mutation in $\mathrm{CC}$ chemokine receptor 5 : studies in populations with contrasting clinical phenotypes, defined racial background, and quantified risk. Mol Med, 3:23-36.

Zingman B, Suleiman J, DeJesus E, et al. 2008. Vicriviroc, a next generation CCCR5 antagonist, exhibits potent, sustained suppression of viral replication in treatment-experienced adults: VICTOR-E1 48-week results [abstract]. 15th Conference on Retroviruses and Opportunistic Infections. Boston, MA. Abstract 39LB. 
